\title{
HOMEOMORPHISMS OF THE UNIT BALL
}

\section{JACK HACHIGIAN ${ }^{1}$}

Let $E^{n}=\left\{x: d(x, 0) \leqq 1, x \in R^{n}\right\}, R^{n}$ is Euclidean $n$-space, $n \geqq 1$. If $T$ and $S$ are any two transformations defined on $E^{n}$ to itself define $\|T-S\|=\sup \left\{d[T(x), S(x)]: x \in E^{n}\right\}$. Consider a continuous transformation $T$ of $E^{n}$ onto $E^{n}$. Denote by $T^{k}$ the $k$ th iterate of $T$, e.g. $T \circ T=T^{2}$. Suppose that $T \neq I$ ( $I$ the identity map) but $T$ has $(n+1)$ fixed points on $\partial E^{n}$. J. Ax [1] has conjectured that under these conditions $\inf _{k}\left\|T^{k}-I\right\|>0$.

Originally, the stronger conclusion $\left\|T^{k+1}-I\right\| \geqq\left\|T^{k}-I\right\|$ was expected to hold for all $E^{n}, n \geqq 1$; however, a counterexample was constructed in [2] for $n \geqq 2$. Note that for $n=1$ this stronger conclusion implies $\inf _{k}\left\|T^{k}-I\right\|>0$ and will be used later.

We first obtain a weaker result (Theorem A) for $n=1,2$ and then use this to prove the main result (Theorem $\mathrm{B}$ ) for $n=1,2$.

A consequence of the main theorem is the known result that an infinite compact zero-dimensional group cannot act effectively on a two-dimensional manifold. The question is open for higher dimensions.

THEOREM A. Let $E^{n}$ be the unit ball in $R^{n}, n=1,2$ and let $T$ be a continuous transformation of $E^{n}$ onto $E^{n}$ such that $T \not \equiv I$, but restricted to $\partial E^{n}, T=I$. Then $\inf _{k}\left\|T^{k}-I\right\|>0$.

Proof. For $n=1$ see [2].

If $T$ is many-one the theorem is trivially true; it is enough therefore to consider a homeomorphism of $E^{2}$ to $E^{2}$.

The rest of the proof is contrapositive. Given $\epsilon>0, \exists N>0$ such that $\left\|T^{k_{i}}-I\right\|<\epsilon$ for all $i>N$. Equivalently,

$$
\sup \left\{d\left[T^{k_{i}}(x), x\right] ; x \in E^{n}\right\}<\epsilon, \quad i>N
$$

which implies

$$
d\left[T^{k_{i}}(x), x\right]<\epsilon \quad \text { for all } x \in E^{2}, \quad i>N .
$$

Let $X$ be a metric space and consider a sequence of maps

$$
g_{1}, g_{2}, \cdots, g_{n}, \cdots
$$

of $X$ into itself. The sequence (2) is called a $C$-sequence if for every

Received by the editors May 22, 1967.

${ }^{1}$ I wish to thank Professor Haskell Cohen for helpful discussions. 
$\epsilon>0, \exists \delta(\epsilon), N(\epsilon)$ such that $d(x, y)<\delta$ implies $d\left[g_{n}(x), g_{n}(y)\right]<\epsilon$ for all $n>N$. As an immediate consequence we note that if (2) is a $C$ sequence, and if each $g_{n}$ is uniformly continuous then (2) is equiuniformly continuous. A result of Edrei [3] now states that if $h$ maps a totally bounded metric space on to itself such that some subsequence

$$
h^{v_{1}}, h^{v_{2}}, \cdots, h^{v_{m}}, \cdots
$$

of $h, h^{2}, h^{3}, \cdots, h^{n}, \cdots$ is a $C$-sequence then $h$ is a homeomorphism and the set of maps $h^{n}[n=0, \pm 1, \pm 2, \cdots]$ is equi-uniformly continuous.

That $\left\{T^{k_{i}}\right\}_{i=1}^{\infty}$ is a $C$-sequence follows at once from

$$
d\left[T^{k_{i}}(x), T^{k_{i}}(y)\right] \leqq d\left[T^{k_{i}}(x), x\right]+d[x, y]+d\left[T^{k_{i}}(y), y\right]
$$

and (1). Hence $\left\{T^{n}\right\}$ is an equi-uniformly continuous set of transformations of $E^{2}$ onto $E^{2}$. This implies $[5$, p. 341] that $T$ is almost periodic. A recent result by Foland [4, p. 1032] states that if $T \mid \partial E^{2}$ $=I$, and $T$ is almost periodic then $T=I$. A contradiction.

Theorem B. Let $E^{n}$ be the unit ball in $R^{n}, n=1,2$ and let $T \neq I$ be $a$ continuous transformation of $E^{n}$ onto $E^{n}$ such that $T$ restricted to $\partial E^{n}$ has $n+1$ fixed points. Then inf $_{k}\left\|T^{k}-I\right\|>0$.

Proof. For $n=1$ Theorem B agrees precisely with Theorem A.

For $n=2$, suppose $\inf _{k}\left\|T^{k}-I\right\|=0$. We know that $T$ has three fixed points, say $x_{1}, x_{2}, x_{3}$, on $\partial E^{2}$. Let $x_{1}, x_{2}, x_{3}$ be arranged in some order say clockwise on $\partial E^{2}$. Define $E_{13}=\left\{x: x \in \partial E^{2}\right.$, and $x$ lies between $x_{1}$ and $\left.x_{3}\right\}$, where the subscripts indicate the points between which the elements of the set lie but the direction always being taken as clockwise. Since a homeomorphism of a compact set takes the boundary onto the boundary, $T$ mapping $E^{2}$ onto $E^{2}$ clearly takes $E_{13}$ on to $E_{13}$. $x_{2} \in E_{13}$ disallows reflection. $T$ restricted to $E_{13}$ therefore satisfies the conditions of Theorem A when $n=1$. Hence $T=I$ on $E_{13}$. Similarly $T=I$ on $E_{31}$ and therefore $T$ must be the identity on all $\partial E^{2}$. The conditions of Theorem A are now satisfied for $\boldsymbol{n}=2$. This completes the proof.

REMARK. Let $D^{2}$ be any simply connected compact subset of $R^{2}$. Let $T: D^{2} \rightarrow D^{2}$ onto, continuous and such that $T \not \neq I, T \mid \partial D^{2}=I$. Then $\inf _{k}\left\|T^{k}-I\right\|>0$.

REMARK. Let $D^{2}$ be any simply connected compact subset of $R^{2}$, homeomorphic to $E^{2}$. Let $f: D^{2} \rightarrow D^{2}$ be onto, continuous, and such that $f$ has three fixed points. Then $\inf _{k}\left\|f^{k}-I\right\|>0$. 


\section{BIBLIOGRAPHY}

1. J. Ax, Communication.

2. H. Cohen and J. Hachigian, On iterates of continuous functions on the unit ball, Proc. Amer. Math. Soc. 18 (1967), 408-411.

3. A. Edrei, On iteration of mappings of a metric space onto itself, J. London Math. Soc. 26 (1951), 96-103.

4. N. E. Foland, A characterization of the almost periodic homeomorphisms on the closed 2-cell, Proc. Amer. Math. Soc. 16 (1965), 1031-1034.

5. W. H. Gottschalk, Minimal sets: an introduction to topological dynamics, Bull. Amer. Math. Soc. 64 (1958), 336-351.

State University of New York at Stony Brook 